Reprod. Nutr. Dévelop., 1983, 23 (2 A), 273-284.

\title{
Etude du comportement sexuel mâle induit par la testostérone chez des brebis adultes ovariectomisées Effet de la dose et de l'expérience sexuelle antérieure
}

\author{
Claude FABRE-NYS, J.-P. SIGNORET, Danielle H. GARNIER \\ avec la collaboration technique de Colette LAVENET, P. ORGEUR, J. C. POIRIER et G. VENIER \\ Station de Physiologie de la Reproduction, I.N.R.A., \\ Nouzilly, 37380 Monnaie, France.
}

Summary. A study of male sexual behaviour induced by testosterone treatment in ovariectomized adult ewes. Effect of different doses and previous sexual experience.

Adult ovariectomized ewes, daily injected with testosterone propionate $(2$ to $22 \mathrm{mg}$ ), showed male-like sexual behaviour, never seen in untreated females, but very similar to that in normal rams. After a few days of latency ( 3 days minimum), this behaviour gradually appeared and increased in frequency until a plateau was reached. The time-course in which the plateau was reached depended on the androgen dose and on previous experience with male sexual behaviour. Some male-like behaviour might have persisted after the end of the treatment.

The results have been discussed in terms of possible mechanisms of action on the central nervous system.

\section{Introduction.}

Quelle que soit l'espèce, ou même le groupe de vertébrés étudié, le comportement sexuel mâle est sous la dépendance de la testostérone (voir revue de Hart, 1974, chez les mammifères mais aussi Balthazart, 1976, chez le canard; Crews et al., 1978, chez le lézard; Moore, 1978, chez le triton). Cependant, le mécanisme de cette action reste obscur. Plusieurs facteurs rendent ces études difficiles, nous en citerons trois :

- Le comportement sexuel mâle évoluant de manière lente (plusieurs jours ou semaines) et progressive (Beach et Holz, 1946), l'effet d'interventions expérimentales est donc malaisé à mesurer.

- L'expérience sexuelle interfère avec la réponse du mâle aux changements de taux hormonaux : un mâle expérimenté conserve une activité sexuelle après castration beaucoup plus longtemps qu'un animal naif (Rosenblatt et Aronson, 1958 ; Manning et Thompson, 1976).

- Aux effets " centraux " des stéroïdes s'ajoutent les effets "périphériques " : les androgènes sont nécessaires au développement des organes copulatoires et peuvent en modifier la sensibilité (Phoenix et al., 1976), ce qui pourra, en retour, 
modifier le déroulement du comportement du mâle (Adler et Bermant, 1966 ; Aronson et Cooper, 1968). L'importance relative de ces deux effets chez un mâle est impossible à déterminer.

C'est pourquoi nous nous sommes proposés d'aborder l'étude du mécanisme d'action de la testostérone en utilisant comme modèle la brebis ovariectomisée adulte.

Chez un tel animal, un traitement par les androgènes provoque l'apparition d'un comportement sexuel mâle, normalement absent chez les femelles de cette espèce (Signoret, 1975). Cette action des stéroïdes est essentiellement centrale puisque l'anatomie génitale est restée de type femelle; en outre, le facteur " expérience » ne peut intervenir.

Nous avons analysé l'effet de traitements prolongés par différentes doses de testostérone, sur la mise en place et le niveau du comportement sexuel mâle de brebis ovariectomisées, en nous intéressant particulièrement à la cinétique de cette action.

\section{Matériel et méthodes.}

Animaux. - Nous avons utilisé des brebis de race lle-de-France, ovariectomisées après la puberté et âgées de 3 à 4 ans. Elles étaient logées en bergerie par groupe unisexué de 10 à 20 animaux.

Traitements. - Terqui et al. (1974) avaient évalué la production journalière d'androgènes testiculaires par des béliers à 10 à $30 \mathrm{mg}$. Par ailleurs, dans un essai préliminaire, Signoret (1975) avait trouvé qu'un traitement par $10 \mathrm{mg}$ par jour de propionate de testostérone était efficace pour induire une activité de type mâle chez des brebis ovariectomisées. Ce traitement a donc été choisi comme base de comparaison.

Compte tenu du rôle possible de l'expérience antérieure et des disponibilités en animaux - qui pouvaient nous conduire à les utiliser dans plusieurs expériences successives - , il était important de connaître la constance des effets obtenus par un même traitement. Pour cette raison, la dose de $10 \mathrm{mg}$ par jour a été employée à plusieurs reprises sur différents animaux ayant déjà subi un lou plusieurs) traitement par les androgènes (19 animaux), ou bien sur des sujets qui $n^{\prime}$ avaient jamais été traités antérieurement (7).

L'effet de la dose d'androgène a ensuite été analysé en administrant respectivement $2 ; 4,5 ; 10$ et $22 \mathrm{mg} /$ jour à 4 groupes de 5 animaux. Ces doses sont telles qu'il existe entre elles une progression géométrique.

Ces traitements ont duré 20 jours dans la première expérience et 52 jours dans la deuxième. Ces périodes correspondent au temps nécessaire à l'obtention d'un niveau d'activité sexuelle stable chez la plupart des animaux.

Dans tous les cas, le stéroïde était dilué dans de l'huile d'olive et administré sous forme d'injections intramusculaires quotidiennes au niveau de l'encolure.

Observations. - Le niveau de comportement sexuel mâle a été évalué au cours d'épreuves comportementales standardisées, d'une durée de $10 \mathrm{~min}$ et faites tous les 3 ou 4 jours. Cette épreuve consistait à introduire chaque animal dans un enclos voisin de son parc d'élevage, où se trouvaient 4 brebis ovariecto- 
misées et maintenues en cycle artificiel (Robinson, 1954), de sorte qu'à chaque épreuve, deux d'entre elles soient réceptives. Les interactions sexuelles décrites chez le bélier (Banks, 1964) sont relevées: flairages de la région ano-génitale, Flehmen (retroussement de la lèvre supérieure à la suite le plus souvent d'un flairage d'urine), approches latérales (mouvements de cour ritualisés) et chevauchements.

Les deux premiers éléments, flairages et flehmen, font également partie du répertoire comportemental des brebis normales mais leur fréquence est beaucoup plus faible que chez les béliers. Approches latérales et chevauchements, par contre, sont caractéristiques de ces derniers.

Nous nous sommes essentiellement intéressés à l'évolution de l'activité sexuelle mâle lors de son apparition et pendant son maintien ; cependant, dans un essai, sa décroissance a été suivie jusqu'à disparition de la présentation régulière des manifestations de type mâle, chez quatre femelles qui avaient reçu $10 \mathrm{mg} /$ jour de propionate de testostérone pendant 20 jours.

Mesure du taux de testostérone circulant. - Des prélèvements de sang ont été effectués tous les 4 jours (avant l'injection journalière, soit $23 \mathrm{~h}$ après la précédente) chez 5 femelles recevant quotidiennement $2 ; 4,5 ; 10$ ou 22 mg de propionate de testostérone (PT).

De plus, pour suivre l'effet à court terme des injections, des prélèvements sériés ont été faits en milieu de traitement soit par raison de commodité, après la $10^{\mathrm{e}}$ injection de $10 \mathrm{mg}$ PT ( $\mathrm{n}=5$ animaux ; moment du prélèvement par rapport à l'injection $=-30 \mathrm{~min},+2 \mathrm{~h} 30$ puis toutes les $2 \mathrm{~h}$ pendant les $12 \mathrm{~h}$ suivantes) et après la 12e injection de $2 ; 4,5$ ou $22 \mathrm{mg}$ de PT ( $\mathrm{n}=5$ animaux ; prélèvements à $-3 \mathrm{~h},+30 \mathrm{~min},+1 \mathrm{~h} \mathrm{30,}+2 \mathrm{~h} \mathrm{30,}+4 \mathrm{~h},+6 \mathrm{~h},+8 \mathrm{~h}$ ).

Enfin, des prélèvements ont été effectués 1, 2, 3, 5, 8, 10, 12, 16, 19, 23 et 39 jours après l'arrêt d'un traitement de 20 jours par $10 \mathrm{mg}$ de PT pour suivre la décroissance du taux de testostérone circulante.

La teneur en testostérone a été mesurée par dosage radioimmunologique directement dans le plasma par la méthode mise au point par Garnier et al. (1978).

Analyse statistique. - Suivant les données dont nous disposions, nous avons employé un test $t$ ou des tests non paramétriques (Siegel, 1956) qui seront précisés dans le texte.

La liaison entre la fréquence d'activité et la durée de traitement ou la dose d'androgène a été déterminée par le calcul de la régression linéaire entre la fréquence et le temps ou la fréquence et la dose.

\section{Résultats.}

Comportement sexuel mâle. - L'administration répétée de propionate de testostérone provoque la mise en place progressive d'un comportement sexuel de type mâle marqué par l'augmentation de la fréquence des flairages anogénitaux et des flehmen et par l'apparition d'approches latérales puis de chevauchements. D'une manière générale, la fréquence moyenne de ces différents actes augmente avec le temps de traitement jusqu'à atteindre un plateau. 
- Effets de traitements successifs. La mise en place du comportement de type mâle et notamment des chevauchements est plus rapide lors d'un deuxième traitement par les androgènes que pendant le premier (fig. $1 a, b ; P \leqslant 0,01$ après 8 jours de traitement et $P \leqslant 0,05$ après 12 ou 20 jours, test de Fisher bilatéral). Cependant, ceci n'est vrai que si le premier traitement avait provoqué l'apparition d'un comportement mâle (fig. 1a, b, c). De même, la fréquence d'activité sexuelle après 20 jours de traitement tend à être supérieure la deuxième fois, quoique les différences ne soient significatives que pour les chevauchements (tabl. 1).

Par contre, aucune modification nette n'est visible entre la deuxième et la septième androgénisation (fig. 1b, d).

-- Effets de la dose d'androgènes. Si l'expérience sexuelle mâle des brebis, au cours de manipulations antérieures est semblable, le comportement de type mâle induit par un nouveau traitement par un androgène dépend de la dose administrée (fig. 2).

(a)
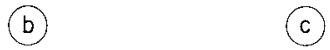

(d)

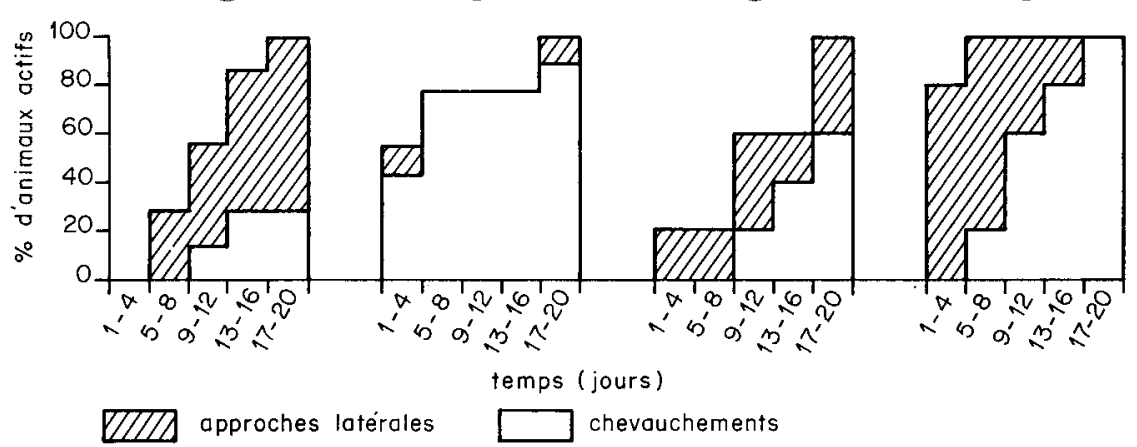

FIG. 1. - Effet de traitements androgènes antérieurs sur la réponse de type mâle à des injections quotidiennes de $10 \mathrm{mg}$ de propionate de testostérone. a : première androgénisation $(\mathrm{n}=7) ; \mathrm{b}$ et $c$ : deuxième androgénisation, les brebis ayant $(b, n=9)$ ou n'ayant pas $(c, n=5)$ présenté de chevauchement à la suite du premier traitement ; $d$ : septième androgénisation $(n=5)$.

\section{TABLEAU 1}

Effet de l'expérience antérieure sur la fréquence d'activité sexuelle mâle de brebis ovariectomisées, après 20 jours de traitement par $10 \mathrm{mg}$ de propionate de testostérone par jour

\begin{tabular}{|c|c|c|c|c|}
\hline & $\begin{array}{l}\text { Approches } \\
\text { latérales }\end{array}$ & $\begin{array}{l}\text { Différents cor } \\
\text { Chevauchements }\end{array}$ & $\begin{array}{l}\text { portements } \\
\text { Flairages } \\
\text { anogénitaux }\end{array}$ & Flehmen \\
\hline $\begin{array}{l}\text { Premier traitement }(7) \\
\text { Deuxième traitement }(9)\end{array}$ & $\begin{array}{l}40,7 \pm 16,6 \\
58,9 \pm 9,1\end{array}$ & $\begin{array}{r}2,9 \pm 2,0 \\
10,3 \pm 2,1^{*}\end{array}$ & $\begin{array}{l}10,1 \pm 2,2 \\
12,4 \pm 3,6\end{array}$ & $\begin{array}{l}1,0 \pm 0,2 \\
0,4 \pm 0,2\end{array}$ \\
\hline
\end{tabular}

* $P \leqslant 0,05$ test $t$.

( ) nombre d'animaux. 

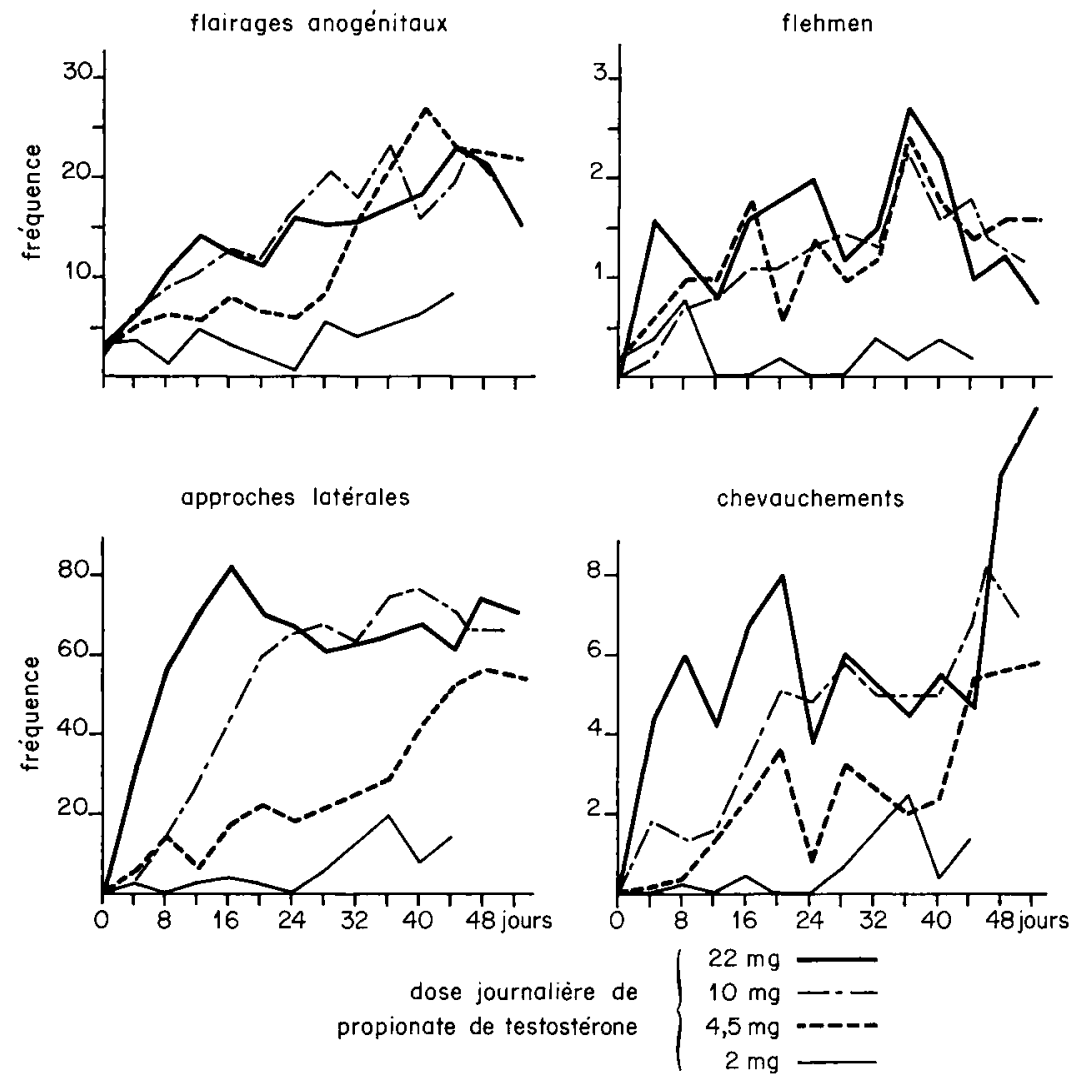

FIG. 2. - Effet de doses croissantes de propionate de testostérone $(2 ; 4,5 ; 10$ et $22 \mathrm{mg}$ par jour) sur la fréquence d'activité sexuelle mâle (fréquence moyenne par animal et par épreuve, $n=5$ dans chanue groupe).

L'apparition des éléments caractéristiques du mâle - approches latérales et chevauchements - est d'autant plus précoce que la dose est plus élevée et il existe une forte corrélation entre la quantité journalière $d$ 'androgène injectée et la vitesse d'augmentation de la fréquence (donnée par la pente de la droite de régression en fonction du temps pendant la période de croissance de l'activité) des approches latérales et des chevauchements $(Y=-0,4 \pm 0,34(+0,01) X$, $r=0,999, P \leqslant 0,01$ pour les approches latérales et $Y=-0,07 \pm 0,037$ $(+0,003) X, r=0,994, P \leqslant 0,05$ pour les chevauchements $)$.

Par contre, le niveau moyen atteint pendant la phase " plateau " est comparable pour les groupes traités par 22 et $10 \mathrm{mg} /$ jour (respectivement $68 \pm 4$ et 65 \pm 3 pour les approches latérales et $6,5 \pm 0,8$ et $5,3 \pm 0,5$ pour les chevauchements). Un niveau non significativement différent est atteint en fin de traitement dans le groupe $" 4,5 \mathrm{mg}$ » $(57 \pm 7$ et $5,6 \pm 1 ; \mathrm{P}>0,1$ test de Mann et Whitney); par contre, la fréquence reste inférieure après traitement par la plus faible dose $(P \leqslant 0,05$ test de Mann et Whitney). 
Pour les flairages, quelle que soit la dose considérée, la progression est continue tout au long du traitement ( $r$ minimum $=0,40, P \leqslant 0,01$ ). Cependant, pour les doses 10 et $22 \mathrm{mg}$, l'augmentation tend à être plus importante au début du traitement. En fin d'expérience, le niveau atteint par les animaux du groupe 4,5 mg n'est pas différent de celui des groupes 10 et $22 \mathrm{mg}$; par contre, il reste inférieur $(P \leqslant 0,05$, test de Mann et Whitney) dans le groupe $2 \mathrm{mg}$.

La fréquence des flehmen n'est pas modifiée dans le groupe traité par la plus faible dose. Dans les autres, elle augmente plus brusquement que celle des flairages puis fluctue autour d'une valeur comparable dans les trois groupes $(1,4 \pm 0,1$ pour $4,5 \mathrm{mg}, 1,5 \pm 0,1$ pour $10 \mathrm{mg}$ et $1,5 \pm 0,2$ pour $22 \mathrm{mg})$.

- Evolution après arrêt d'un traitement. La disparition du comportement de type mâle à l'arrêt d'un traitement de 20 jours par $10 \mathrm{mg}$ de propionate de testostérone s'effectue en deux temps (tabl. 2). Pendant la première phase, l'activité diminue brutalement : 4 jours après l'arrêt du traitement, la fréquence moyenne des chevauchements a diminué de $96 \%$, celle des approches latérales de $90 \%$, celle des flairages de $60 \%$ et les flehmen déjà rares dans ce groupe ont disparu. Cette première décroissance est due à une diminution du niveau de comportement sexuel de chaque animal lau $8^{e}$ jour, ils présentent encore tous des approches latérales). Une activité faible et irrégulière se maintient ensuite pendant la deuxième phase, les chevauchements disparaissant avant les approches latérales. Dans certains cas, des chevauchements occasionnels ont été observés chez des femelles, 4 mois après l'arrêt d'un traitement de 70 jours par $10 \mathrm{mg}$ de propionate de testostérone.

Comportement sexuel femelle. - Simultanément à son effet sur le comportement de type mâle, un traitement prolongé par la testostérone provoque une réceptivité sexuelle femelle, visible quand la brebis est mise en contact avec un bélier sexuellement actif. L'acceptation du chevauchement apparaît plus rapidement que l'activité sexuelle mâle ( $24 \mathrm{~h}$ après la première injection) et persiste, de manière plus ou moins régulière pendant tout le traitement sauf dans le cas de la plus petite dose (fig. 3). Cependant, ces données, limitées à l'acceptation du chevauchement, ne nous permettent pas d'affirmer que le comportement sexuel femelle induit est identique à celui de femelles normales pendant l'œestrus.

\section{Testostérone plasmatique}

- Effets des différentes doses. L'évolution du taux de testostérone plasmatique au cours d'un traitement prolongé par $2 ; 4,5$ ou $10 \mathrm{mg}$ de propionate de testostérone par jour est similaire (fig. 4). Elle est caractérisée par une augmentation significative dès le premier prélèvement. Au cours d'une expérience préliminaire, nous avions observé que $2 \mathrm{~h} 30$ après la première injection de $10 \mathrm{mg}$, le taux plasmatique de testostérone atteignait des valeurs comprises entre 5 et 10 $\mathrm{ng} / \mathrm{ml}$ suivant les animaux. Ensuite le taux de testostérone se maintient à un niveau stable pendant le reste de l'expérience. Par contre, dans le groupe recevant $22 \mathrm{mg} /$ jour, ce taux continue à augmenter avec le temps.

Le taux moyen de testostérone plasmatique de chaque animal, calculé pour l'ensemble de l'expérience dans les quatre groupes est fortement lié à la dose journalière administrée $(r=0,963, P<0,001)$. 


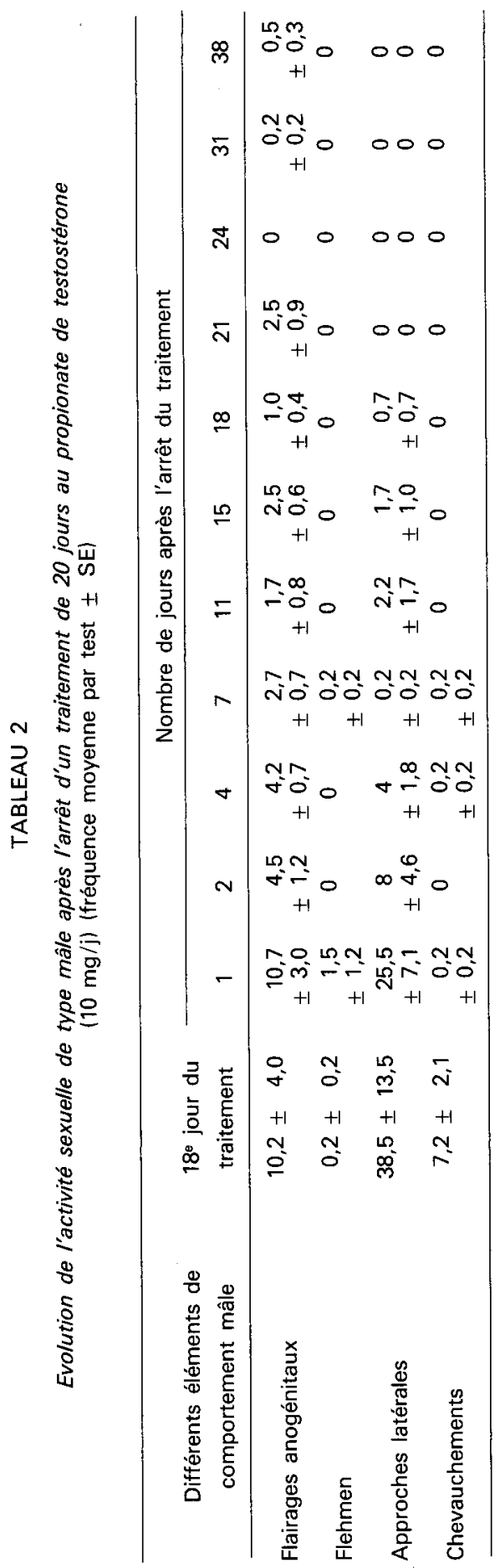



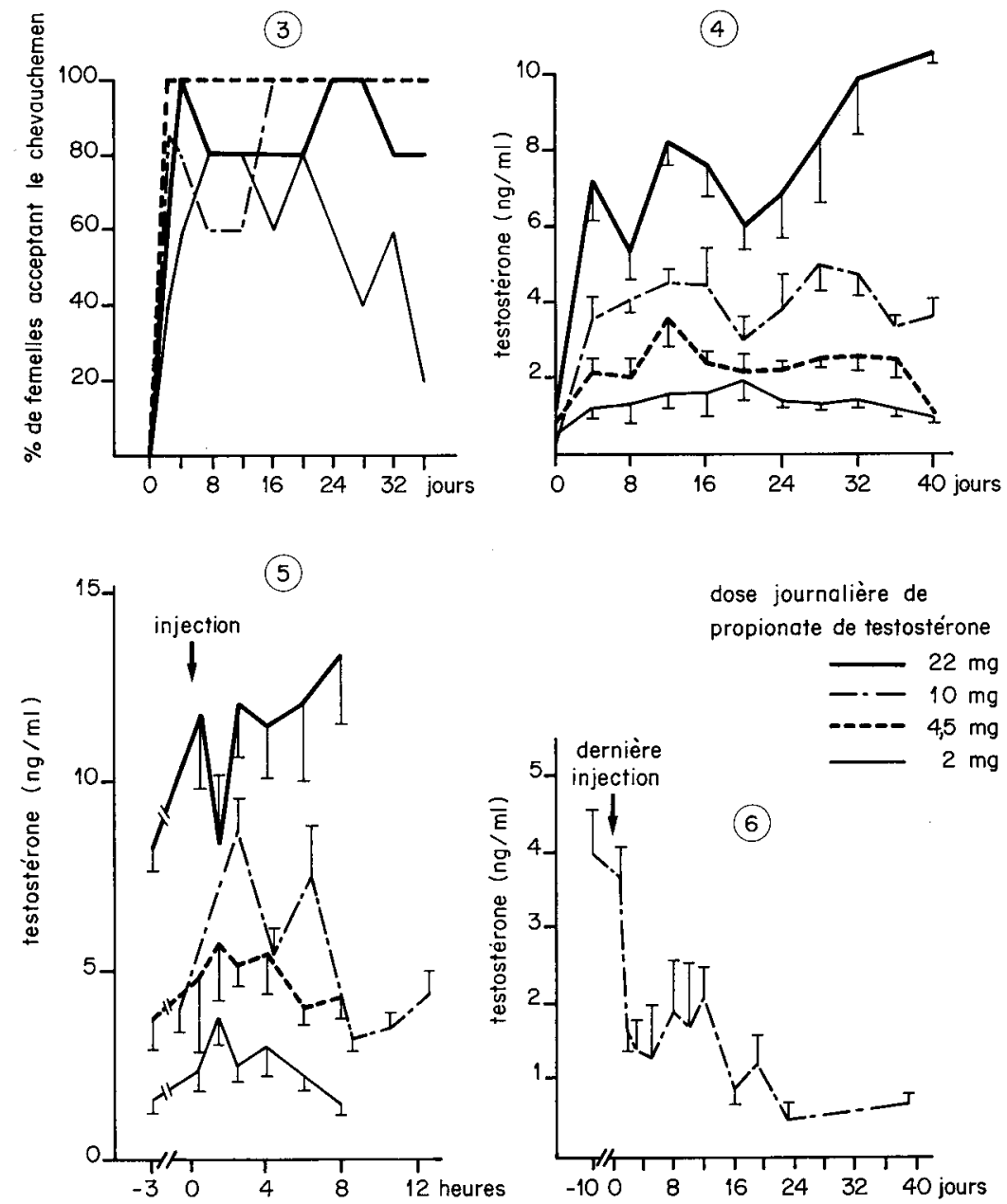

FIG. 3. - Effet de doses croissantes de propionate de testostérone $(2 ; 4,5 ; 10$ et $22 \mathrm{mg}$ par jour) sur la réceptivité sexuelle femelle ( $\mathrm{n}=5$ dans chaque groupe).

FIG. 4. - Evolution du taux de testostérone plasmatique au cours du traitement quotidien par différentes doses de propionate de testostérone $(2 ; 4,5 ; 10$ et $22 \mathrm{mg}, \mathrm{n}=\mathbf{5}$ dans chaque groupe, valeurs moyennes $\pm \mathrm{SE}$ ).

FIG. 5. - Evolution du taux de testostérone plasmatique après l'administration de la dixième (groupe recevant la dose $10 \mathrm{mg}$ ) ou de la douzième (groupe recevant $2 ; 4,5$; et $22 \mathrm{mg}$ ) injection journalière de propionate de testostérone ( $\mathrm{n}=5$ dans chaque groupe, valeurs moyennes $\pm \mathrm{SE}$ ).

FIG. 6. - Evolution du taux de testostérone plasmatique après l'arrêt d'un traitement de 20 jours par $10 \mathrm{mg}$ par jour de propionate de testostérone ( $\mathrm{n}=4$, valeurs moyennes $\pm \mathrm{SE}$ ).

Excepté pour la plus forte dose, le profil journalier (fig. 5) apprécié par les prélèvements effectués pendant les heures suivant la $10^{\mathrm{e}}$ (dose $10 \mathrm{mg}$ ) ou la $12^{\mathrm{e}}$ injection (doses 2, 4,5 et $22 \mathrm{mg}$ ) comporte un pic durant environ six heures 
après lequel est atteinte une valeur comparable à celle mesurée immédiatement avant l'injection ou dans les échantillons prélevés tous les 3 ou 4 jours.

- Evolution après arrêt du traitement. Vingt-quatre heures après la dernière injection journalière de $10 \mathrm{mg}$ de propionate de testostérone (dans une série en comportant 20), le taux de testostérone plasmatique est semblable au " taux de base " observé pendant le traitement. II diminue de manière importante pendant les $24 \mathrm{~h}$ suivantes, puis plus lentement pour atteindre $0,5 \mathrm{ng} / \mathrm{ml} 3$ semaines après ; ce taux est retrouvé 40 jours après l'arrêt du traitement (fig. 6).

Relation entre le taux de testostérone plasmatique et le comportement sexuel. La vitesse d'augmentation de la fréquence des approches latérales et des chevauchements d'un groupe est liée à la dose journalière qu'il reçoit et au taux moyen de testostérone dans ce groupe $(r=0,998, P<0,001$ pour les approches latérales et $r=0,992, P<0,01$ pour les chevauchements).

D'autre part, le taux moyen de testostérone plasmatique d'un animal est lié à la fréquence moyenne de chacun de ses éléments de comportement sexuel, si la comparaison porte sur l'ensemble des animaux $(n=20)$. Suivant les comportements, la valeur du coefficient de corrélation $r$ varie de 0,54 à 0,66 et la liaison est toujours significative ( $P<0,05$ ou $P<0,01$ suivant les cas). Par contre, aucune liaison n'apparaît si nous comparons différents individus d'un même groupe ou bien des jours successifs chez un même animal.

\section{Discussion.}

La plupart des caractéristiques de la relation entre les hormones et le comportement sexuel mâle, établies chez le mâle (Beach et Holz, 1946 ; Clegg et al., 1969 ; Mattner, 1977) sont retrouvées chez les brebis ovariectomisées et traitées par les androgènes à l'âge adulte : nous observons ainsi un retard des modifications de l'activité sexuelle par rapport aux changements de concentration de la testostérone plasmatique, l'existence d'une relation entre la dose d'androgène et la réponse comportementale pendant que la fréquence d'activité augmente, la présence d'un niveau maximum d'activité sexuelle indépendant de la dose et enfin, la persistance de manifestations sexuelles mâles après l'arrêt d'un traitement.

La situation particulière des femelles ovariectomisées à l'âge adulte (anatomie génitale, naïveté quant à l'exercice du comportement mâle, etc...l fait que les effets observés sont dûs essentiellement à l'action activatrice (par opposition à l'action organisatrice prénatale) centrale de la testostérone ce qui les rend particulièrement intéressants.

Ces résultats montrent que les stimulations d'origine génitale - limitées par l'anatomie restée de type femelle - et l'éventuelle masculinisation des structures cérébrales pendant le développement, ont une importance mineure dans le contrôle hormonal du comportement sexuel mâle chez les Ovins. Ceci confirme l'analyse que nous avions faite sur la qualité de ce comportement, montrant que les femelles étaient capables de présenter tous les éléments du comportement sexuel du mâle, y compris une réaction ressemblant à celle présentée par le bélier lors de l'éjaculation (Fabre, 1977). 
De plus, la lenteur avec laquelle l'action de la testostérone se manifeste apparaît comme un phénomène remarquable, particulier au comportement mâle et se situant au niveau du système nerveux central.

Quelques heures après la première injection de propionate de testostérone à des brebis ovariectomisées, le taux plasmatique de cette hormone est élevé. Vingt-quatre heures plus tard, ces animaux présentent une réceptivité sexuelle femelle mais il faut attendre au minimum 3 jours pour voir apparaître les premières manifestations de comportement sexuel mâle. Chez le bélier, Rouger (1974) et Schanbacher et Lunstra (1976) ont montré que l'augmentation saisonnière de testostérone précèdait de plusieurs semaines celle de l'activité sexuelle.

Ceci suppose la nécessité de la mise en place d'un système différent de celui impliqué dans la régulation de l'activité sexuelle femelle lou d'un fonctionnement différent), les deux systèmes pouvant être actifs en même temps.

De plus, malgré les nombreux intermédiaires - nerveux et biochimiques existant certainement entre la présence de l'hormone et un changement de comportement, il existe une liaison forte entre la dose d'androgène et l'effet observé (vitesse d'apparition ou fréquence moyenne d'un comportement), ceci jusqu'à un niveau " plateau " à partir duquel le système semble " saturé ». II faut noter également que cette relation effet-dose n'est pas constante ; notamment il n'est pas possible de relier les variations de niveau de comportement d'un individu à celles de son équilibre hormonal. Ces observations sont comparables à celles de nombreux auteurs étudiant des mâles entiers (Harding et Feder, 1976 ; D'Occhio et Brooks, 1976 ; Mattner, 1977). La lenteur de la manifestation de l'effet de la testostérone qui est à l'origine du décalage entre les variations de concentration hormonale et leur action pourrait expliquer qu'il existe à la fois une dépendance et une certaine indépendance entre le taux d'hormone circulant et l'intensité du comportement. Ainsi, la réponse dépend de la dose quand la comparaison porte sur un effet global des androgènes comme lorsque l'analyse porte sur la fréquence moyenne d'activité d'animaux ayant subi des traitements nettement différents. C'est le cas dans nos expériences ou lorsque l'activité de béliers intacts pendant la saison sexuelle est comparée à celle de béliers castrés ou de béliers intacts hors de la saison de reproduction. Par contre, chez des femelles traitées comme chez le mâle intact, il n'est pas possible de relier les variations journalières de niveau d'activité sexuelle à celles du taux d'androgènes.

Enfin, l'exercice même du comportement de type mâle par un animal, quel que soit son sexe, modifie ses capacités ultérieures à présenter ce comportement. Dans nos expériences, I'apparition des éléments de type mâle est plus lente chez des brebis naïves que chez des femelles expérimentées; de même, ces dernières peuvent présenter de manière sporadique, indépendamment de tout traitement, des éléments de comportement de type mâle, alors que la femelle normale ne le fait pas.

Chez les ovins, alors que la sexualisation par des androgènes pendant la vie fœtale est peu importante pour le développement de l'activité sexuelle mâle (Fabre, 1977), une maturation plus tardive des mécanismes impliqués dans cette activité est possible. Contrairement aux modifications qui ont lieu pendant la vie 
fœetale, l'imprégnation hormonale seule ne peut pas provoquer cette maturation tardive. Une participation active de l'animal : la réalisation d'un comportement de type mâle est nécessaire, ce qui apparente cette maturation à un processus d'apprentissage.

Reçu en juillet 1982.

Accepté en novembre 1982.

\section{Références}

ADLER N., BERMANT G., 1966. Sexual behavior of male rats : effects of reduced sensory feedback. J. comp. Physiol. Psychol., 61, 240-243.

ARONSON L. R., COOPER M. L., 1968. Desensitization of the glans penis and sexual behavior in cats, 51-82. In DIAMOND M., Perspectives in reproduction and sexual behavior. Indiana Univ. Press, Bloomington.

BANKS E. M., 1964. Some aspects of sexual behaviour in domestic sheep, Ovis aries. Behaviour, 23, 249-279.

BALTHAZART J., 1976. Daily variations of behavioural activities and of plasma testosterone levels in the domestic duck Anas platyrhynchos. J. Zool. London, 180, 155-173.

BEACH F. A., HOLZ A. M., 1946. Mating behavior in male rat castrated at various age and injected with androgen. J. exp. Zool., 101, 91-142.

CLEGG M. T., BEAMER W., BERMANT G., 1969. Copulatory behavior of the ram, Ovis aries. III : Effects of pre- and post-puberal castration and androgen replacement therapy. Anim. Behav., 17, 712-717.

CREWS D., TRAINA V., WETZEL F. T., MULLER C., 1978. Hormonal control of male reproductive behavior in the lizard, Anolis carolinensis : role of testosterone, dihydrotestosterone and estradiol. Endocrinology, 103, 1814-1821.

D'OCCHIO M. J., BROOKS D. E., 1976. The influence of androgens and oestrogens on mating behaviour in male sheep. Theriogenology, 6, 614.

FABRE C., 1977. Existence of an ejaculatory-like reaction in ewes ovariectomized and treated with androgens in adulthood. Horm. Behav., 9, 150-155.

GARNIER D. H., COTTA Y., TERQUI M., 1978. Androgen radioimmunoassay in the ram : results of direct plasma testosterone and dehydroepiandrosterone measurement and physiological evaluation. Ann. Biol. anim. Biochim. Biophys., 18, 265-281.

HARDING C. F., FEDER H. H., 1976. Relation between individual differences in sexual behavior and plasma testosterone levels in the guinea pig. Endocrinology, 98, 1198-1205.

HART B. J., 1974. Gonadal androgen and sociosexual behavior of male mammals : a comparative analysis. Psychol. Bull., 81, 383-400.

MANNING A., THOMPSON M. L., 1976. Post-castration retention of sexual behaviour in the male BDF $_{1}$ mouse : the role of experience. Anim. Behav., 24, 523-533.

MATTNER P. E., 1977. Seasonal variation in libido of intact merino rams and of testosteronetreated castrates. Theriogenology, 8, 149.

MOORE F. L., 1978. Differential effects of testosterone plus dihydrotestosterone on male courtship of castrated newts, Taricha granulosa. Horm. Behav., 11, 202-208.

PHOENIX C. H., COPENHAVER K. H., BRENNER R. M., 1976. Scanning electron microscopy of penile papillae in intact and castrated rat. Horm. Behav., 7, 217-227.

ROBINSON T. J., 1954. Relationship of oestrogen and progesterone in oestrus behaviour of the ewe. Nature, London, 173, 878.

ROSENBLATT J. S., ARONSON L. R., 1958. The decline of sexual behavior in male cats after castration with special reference to the role of prior sexual experience. Behaviour, 12, 285338.

ROUGER Y., 1974. Etude des interactions de l'environnement et des hormones sexuelles dans la régulation du comportement sexuel des Bovidae. Th. Doct. Sci. nat., Rennes, $197 \mathrm{pp}$. 
SCHANBACHER B. D., LUNSTRA D. D., 1976. Seasonal changes in sexual activity and serum levels of LH and testosterone in Finnish Landrace and Suffolk rams. J. anim. Sci., 43, 644650.

SIGNORET J. P., 1975. Effects of oestrogen and androgen on the sexual behaviour responses of the ovariectomized ewe. Psychoneuroendocrinology, 1, 179-184.

SIEGEL S., 1956. Non parametric statistics for behavioral sciences. Mc Graw Hill Book Co., New York.

TEROUI M., GARNIER D. H., COTTA Y., ROUGER Y. A., 1974. Dynamics of testosterone and oestradiol-17 $\beta$ concentrations in sheep. J. Steroid Biochem., 5, 379. 